\title{
The Effect of Thermal Parameters on the Flow Temperature of a Magnetized Plasma in a Sphere
}

\author{
Tuduo Biepremene Sebastian ${ }^{1, ~ *}$, Abbey Tamunoimi Michael ${ }^{1}$, Alagoa Kingsley D. ${ }^{2}$, \\ Onwuneme Sylvester Ebere ${ }^{1}$
}

${ }^{1}$ Department of Physics, University of Port Harcourt, Port Harcourt, Nigeria

${ }^{2}$ Department of Physics, Niger Delta University, Amassoma, Nigeria

\section{Email address:}

biepremene_tuduo@uniport.edu.ng (Tuduo B. S.), tamunoimi.abbey@uniport.edu.ng (Abbey T. M.), dalagoa@yahoo.com (Alagoa K. D.), ebere.onwuneme@uniport.edu.ng (Onwuneme S. E.)

${ }^{*}$ Corresponding author

\section{To cite this article:}

Tuduo Biepremene Sebastian, Abbey Tamunoimi Michael, Alagoa, Kingsley D., Onwuneme Sylvester Ebere. The Effect of Thermal Parameters on the Flow Temperature of a Magnetized Plasma in a Sphere. International Journal of Astrophysics and Space Science. Vol. 7, No. 3, 2019, pp. 30-35. doi: 10.11648/j.ijass.20190703.12

Received: July 19, 2019; Accepted: August 29, 2019; Published: September 18, 2019

\begin{abstract}
The effect of thermal parameters on the flow temperature of a magnetized plasma in a sphere was studied. The study models astrophysical environments such as the Sun, which have spherical outline. The governing equations of the problem were obtained based on the Navier-Stokes equations under the Boussinesq's approximation. The solutions to the resulting equations were sought by means of the general perturbation method and the results were graphically represented with radial distance, $r=1.0$ on the figures corresponding to the surface of the sphere. The thermal parameters; particularly, the radiation parameter, $N^{2}$ and free convection parameter, $G r$. were investigated in this study with a view to determine the effect of varying these parameters on the plasma flow temperature. Increasing both $N^{2}$ and $G r$. led to a decrease in the plasma flow temperature in the sphere. However, above the sphere (i.e. at radial distances, $r>1.0$ ) where the plasma density is sparse, increasing $N^{2}$ and $G r$. produced a corresponding increase in the plasma flow temperature. The decrease in the plasma flow temperature within the sphere with increase in the thermal parameters was observed to be more significant between radial distances, $r=0.25$ and $r=0.7$ (i.e., $0.25 \leq r \leq 0.7$ ) than between $r=0.7$ and $r=1.0$ (i.e., $0.7 \leq r \leq 1.0$ ). This is attributable to the prevalence of partially ionized heavy elements within $0.7 \leq r \leq 1.0$ (corresponding to the convection zone of the solar interior) which trap the high energy photons thereby reducing the rate of radiative heat loss.
\end{abstract}

Keywords: Thermal Parameters, Flow Temperature, Magnetized Plasma

\section{Introduction}

The Sun ispowered with the ability to replenish the huge amount of energy lost to solar space from the nuclear fusion process at the core. Of the very high temperature of about 1.5 $\mathrm{x} 10^{7} \mathrm{~K}$ at the core of the Sun, the temperature at the solar surface is only about $5,780 \mathrm{~K}$. It therefore means that most of the energy is lost on the way to the solar surface. According to the studies [1-3], bodies with high core temperatures are abound in astrophysical environments. And the high temperature differential between the core and surface of such bodies induce radiative and convective heat transfer. That is, energy transmission through the plasma of the solar interior is mainly by means of radiation and convection [4]. This suggests that the dynamics of radiation and convection allow for the progressive reduction in the transmitted energy.

Heat transfer mechanisms are of high significance in electrically conducting and magnetized fluid flow problems commonly encountered in astrophysics and engineering. Several works have been done in the literature that borders on heat transfer problems. In their study on the flow of a two-component plasma model in a porous rotating sphere investigated the effect of radiation on the temperature distribution in the interior of astrophysical bodies [5]. Whereas as the study [6] investigated the flow of a low density thermally radiating two-component plasma in the presence of mass transfer and Hall current; while, the study [7] studied a two-component plasma model with 
radiative heat transfer past a slowly roatating porous hot sphere under an optically thin gas approximation. Also, [8] studied the effect of slip parameter on hydromagnetic oscillatory flow combined with heat and mass transfer. Much work has equally been done in the literature that incorporated radiative and convective heat transfer $[1,5,7,9]$, as applicable in stellar interiors.

In the studies above the plasma density was considered constant; that is, the effect of plasma compressibility assumed negligible. But, the statistics presented by the researcher [10] shows that the plasma density within the solar sphere and atmosphere vary with radial distance from the core. The study [11] have also shown that ignoring the effect of compressibility is dangerous. This is because experiment indicates that free convection motion in a flow regime is usually caused by changes in the local density due to variations in the hydrostatic pressure resulting in isotropic acceleration of the fluid. The variability of solar plasma density was also acknowledged by the researches [12, 13] in their studies to investigate the effect of gravity on the stability of solar plasma and the temperature distribution in the solar sphere, respectively. In this study therefore, we considered an exponential representationof the solar plasma density.

\section{The Physics of the Problem and the Governing Equations}

The problem models the flow temperature of a magnetized plasma in a sphere, by considering an exponential varying density of the form;

$$
\begin{gathered}
\rho^{\prime}(r)=\rho_{0} e^{-\alpha_{0} r} \\
\rho^{\prime}\left[\left(V^{\prime} \bullet \nabla\right) V^{\prime}\right] \\
\rho^{\prime} C_{p}\left[(V \bullet \nabla) T^{\prime}\right]=K \nabla^{2} T^{\prime}-\nabla q_{r}^{\prime}
\end{gathered}
$$$$
\rho^{\prime}\left[\left(V^{\prime} \bullet \nabla\right) V^{\prime}\right]=-\nabla \bullet P^{\prime}+\mu \nabla^{2} V^{\prime}-\frac{\mu}{\chi} V^{\prime}-\sigma B_{0}^{2} V^{\prime}+\rho^{\prime} \beta g\left(T^{\prime}-T_{w}\right)
$$

and

$$
\nabla \bullet \nabla q_{r}^{\prime}-3 \sigma \varepsilon_{r}^{2} q_{r}^{\prime}-4 \sigma \varepsilon_{r} T^{\prime 3} \nabla T^{\prime}=0
$$

where, $\alpha_{0}$, the density parameter; and $\rho_{0}$, dimensionless density; $g$, the accelerations due to gravity and the superscript (') indicates dimensional variable. Equations (3) to (6) are respectively the continuity, momentum, heat distribution and the generalized Rosseline radiative heat transfer flux.

As in the case $[6,12]$, the plasma gases in the intergalactic and interplanetary layers are seen to be rarefied. The plasma optical property, $\varepsilon_{r}$ in such case is far less than one (i.e., $\varepsilon_{r}$ $\ll 1)$, so that the gas in these region are mostly regarded as optically thin. Hence, the generalized Rosseline radiative heat flux integro-differential equation for the optically thin limit can be expressed as;

$$
T^{\prime}=T_{\infty}+\phi
$$

and magnetic field of the form;

$$
B=B_{0}+B^{\prime}
$$

If the sphere is allowed to rotate slowly about the azimuthal with an angular velocity, $\Omega_{0}$; itsmagnetic Reynolds number, $R_{m}$ would be far less than 1 (i.e. $R_{m} \quad 1$ ), such that the induced magnetic field $B^{\prime}$ can be neglected, leaving only the applied magnetic field $B_{0}$ toinfluence the flow.

Therefore, if $\rho^{\prime}\left(r^{\prime}\right)$, the plasma density as a function of radial distance, $r^{\prime} ;\left(u^{\prime}, v^{\prime}, w^{\prime}\right)$, the velocity components in the orthogonal $\left(r^{\prime}, \theta, \varphi\right)$-directions, respectively of the spherical coordinate system; $p^{\prime}$, the pressure; $T^{\prime}$, the temperature; $q_{r}^{\prime}$, the radiative heat flux vector; $\mu$, the dynamical viscosity; $\chi$, the permeability; $C_{p}$, the specific heat capacity at constant pressure; $\kappa$, the thermal conductivity; $\sigma$, the Stefan-Boltzmann constant; $\gamma$, thermal expansivity; $\varepsilon_{r}$, the absorption coefficient and $T_{\infty}$, the wall temperature of the medium at equilibrium with $T_{w}$, the wall temperature which was kept constant so that temperature differential is large enough to warrant radiation or radiative heat transfer, then the governing equations based on the Navier-Stokes equations under the Boussinesq approximation, following studies $[1,5,7,14,15]$ are presented in dimensional forms as follows;

$$
\nabla \bullet\left(\rho^{\prime} V^{\prime}\right)=0
$$

$$
\nabla q_{r}^{\prime}=16 \varepsilon_{r} \sigma\left(T^{\prime 4}-T_{\infty}^{4}\right)
$$

Furthermore, from the statistical data presented by [12], as well as the models of $[1,15]$, the temperature difference between adjacent layers of the plasma is not much compared to each other, thus;

where, $\phi$ is a small temperature correction factor, such that, $O\left(T^{\prime}\right) \gg>\phi>O\left(T_{\infty}\right)$, then Equation (7) reduced to;

$$
\nabla q_{r}^{\prime}=16 \sigma \varepsilon_{r} T_{\infty}^{3}\left(T_{\infty}+\phi\right)
$$

such that the heat transfer equation becomes;

$$
\rho^{\prime} C_{p}\left[\left(V^{\prime} \bullet \nabla\right) \phi\right]=K \nabla^{2} \phi-16 \sigma \varepsilon_{r} T_{\infty}^{3} \phi
$$

Subjecting Equations (3), (4) and (10) to the following 
boundary conditions;

$$
T^{\prime}=T_{c} ; u^{\prime}, v^{\prime}, w^{\prime}=0 ; T^{\prime}=T_{w}
$$

then introducing the following non-dimensional relations;

$$
\begin{gathered}
r=\frac{r^{\prime}}{R_{0}},(u, v, w)=\frac{\left(u^{\prime}, v^{\prime}, w^{\prime}\right)}{\Omega_{0} R_{0}}, \rho(r)=\frac{\rho^{\prime}(r)}{\rho_{0}}, \Theta=\frac{\phi-T_{\infty}}{T_{w}-T_{\infty}} \\
\operatorname{Re}=\frac{\rho_{0} \Omega_{0} R_{0}^{2}}{\mu}
\end{gathered}
$$

the rotational Renolds number,

$$
\chi^{2}=\frac{R_{0}^{2}}{k}
$$

the porosity parameter,

$$
\begin{gathered}
M^{2}=\frac{\sigma B_{0}^{2} R_{0}^{2}}{\mu} \\
\rho \frac{\partial u}{\partial r}+\frac{2 \rho u}{r}+u \frac{\partial \rho}{\partial r}+\frac{\rho v}{r} \cot \theta+\frac{\rho}{r} \frac{\partial v}{\partial \theta}=0 \\
\operatorname{Re} \rho\left[u \frac{\partial u}{\partial r}+\frac{v}{r} \frac{\partial u}{\partial \theta}-\frac{v^{2}+w^{2}}{r}\right]=\left[\nabla^{2}-\chi^{2}-M^{2}-\frac{1}{r^{2} \sin ^{2} \theta}\right] u-\frac{2}{r^{2}} \frac{\partial v}{\partial \theta}-\frac{2 v}{r^{2}} \cot \theta-\rho G_{r} \Theta \\
\operatorname{Re} \rho\left[\frac{\partial v}{\partial t}+u \frac{\partial v}{\partial r}+\frac{v}{r} \frac{\partial v}{\partial \theta}+\frac{u v}{r}-\frac{w^{2}}{r} \cot \theta\right]=\left[\nabla^{2}-\chi^{2}-M^{2}-\frac{1}{r^{2} \sin ^{2} \theta}\right] v+\frac{2}{r^{2}} \frac{\partial u}{\partial \theta}+\rho G_{r} \Theta \\
\operatorname{Re} \rho\left[u \frac{\partial w}{\partial r}+\frac{v}{r} \frac{\partial u}{\partial \theta}+\frac{u w}{r}+\frac{v w}{r} \cot \theta\right]=\left[\nabla^{2}-\chi^{2}-M^{2}-\frac{1}{r^{2} \sin ^{2} \theta}\right] w+\rho G_{r} \Theta
\end{gathered}
$$$$
N^{2}=\frac{16 \alpha_{r} \sigma R_{0}^{2} T_{\infty}}{\kappa}
$$

the radiation or heat transfer parameter.

and eliminating the pressure gradients in the $r$ and $\theta$-directions the governing equations in spherical coordinate system $(r, \theta, \varphi)$ for the flow symmetrical about $\varphi$-axis, become;

$$
\operatorname{Re} \operatorname{Pr} \rho\left[u \frac{\partial \Theta}{\partial r}+\frac{v}{r} \frac{\partial \Theta}{\partial \theta}\right]=\left(\nabla^{2}-N^{2}\right) \Theta
$$

\section{Method of Solution}

Finding analytical solutions for problems in the physical sciences and engineering has always been a difficult task for researchers. This is because most of the equations that arise from such problems are usually non-linear such that solutions are not easy to obtain [16-19]. Generally, asymptotic solutions are sought for problems with such complex equations [19].

The non-dimensionalized equations above are highly nonlinear and coupled, and as such are intractable. Therefore, we adopt the general perturbation method to obtain approximate solutions for the governing equations by setting up a perturbation power series about the Reynolds number, $R e$, since the sphere was allowed to rotate slowly. Thus, the solution to each of the flow variables is expressed in the form;

$$
f(r, \theta)=f_{0}(r, \theta)+\operatorname{Re} f_{1}(r, \theta)+\operatorname{Re}^{2} f_{2}(r, \theta)+\operatorname{Re}^{3} f_{3}(r, \theta)+---
$$

However, for a sufficiently small $\operatorname{Re}($ i.e. $O(\operatorname{Re})<<1$ ), the higher-order terms of Equation (16) will make negligible additions to the solution. Therefore, ignoring the higher order terms, we truncate the perturbation series after first order correction term;

$$
f(r, \theta)=f_{0}(r, \theta)+\operatorname{Re} f_{1}(r, \theta)
$$

The solutions to the resultingorders $O\left(f_{0}\right)$ and $O\left(f_{1}\right)$ equations for each of the flow variables are effected by using the following transformations;

$$
\begin{gathered}
\Theta_{0}(r, \theta)=\Theta_{0}(r) \sin \theta \\
w_{0}(r, \theta)=w_{0}(r) \sin \theta \\
u_{0}(r, \theta)=u_{0}(r) \cos \theta \\
v_{0}(r, \theta)=v_{0}(r) \sin \theta \\
\Theta_{1}(r, \theta)=\Theta_{1}(r) \sin 2 \theta
\end{gathered}
$$




$$
\begin{gathered}
w_{1}(r, \theta)=w_{1}(r) \sin 2 \theta \\
u_{1}(r, \theta)=u_{1}(r)\left(2 \cos ^{2} \theta-\sin ^{2} \theta\right)
\end{gathered}
$$

and

$$
v_{1}(r, \theta)=v_{1}(r) \sin 2 \theta
$$

and the results obtained are graphically presented using the Wolfram Mathematica computing software (Mathematic 9).

\section{Results and Discussion}

This section presents the results and discussion of findings. Figure 1, shows the temperature profile of the solar sphere, where the point 1.0 corresponds to the solar surface. While, Figures 2 and 3 show the temperature profiles for various values of radiation parameter, $N^{2}$ and free convection parameter, $G_{\mathrm{r}}$, respectively. For small values of radiation parameter, $N^{2}$, as indicated in Figure 2, increase in $N^{2}$ leads to a reduction in temperature of the plasma.

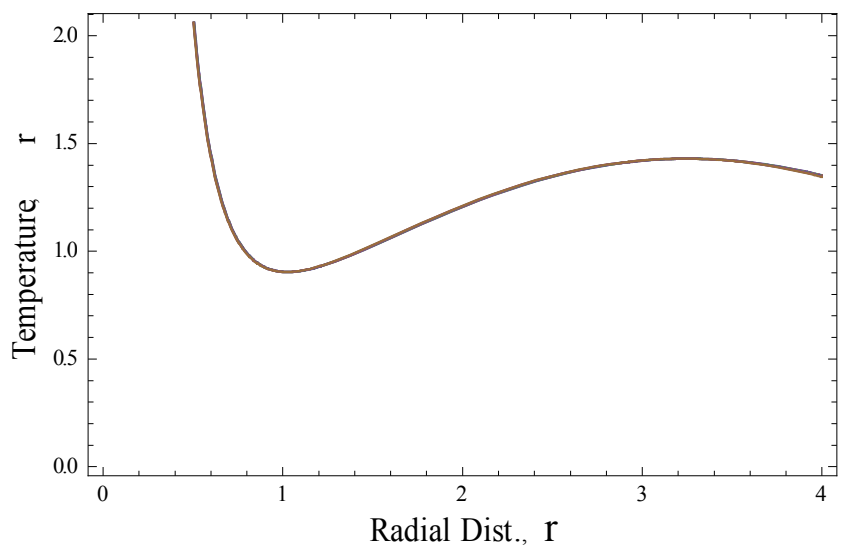

Figure 1. A Plot of Temperature, $\Theta(r)$ against Radial Distance, $r$ : Showing Plasma Flow Temperature Profile.

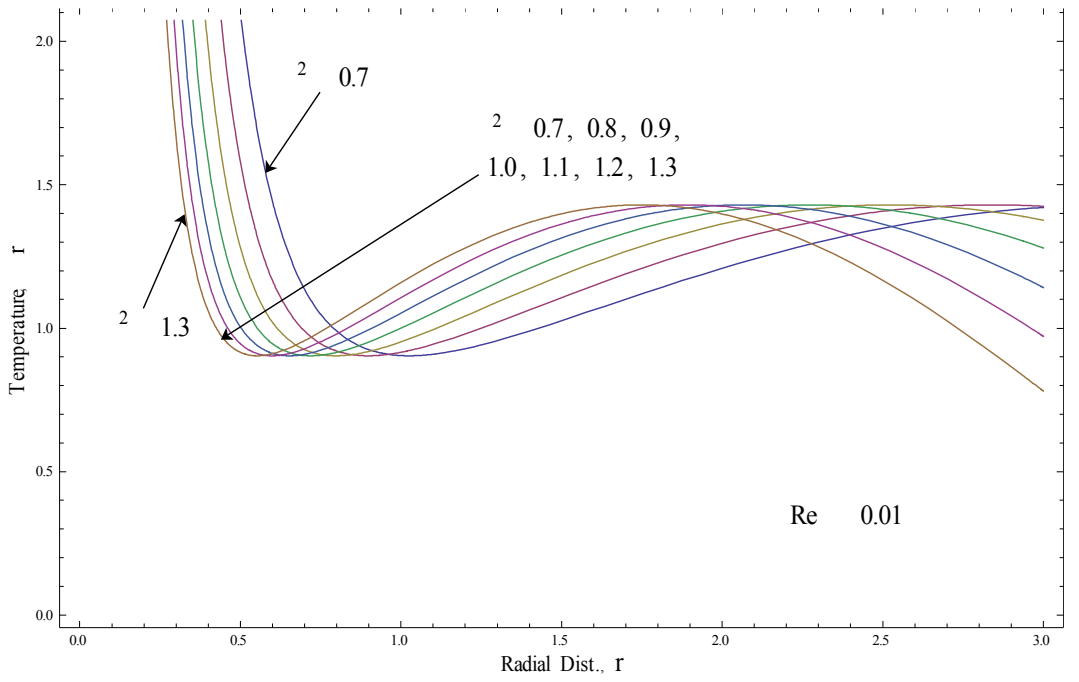

Figure 2. A Plot of Temperature, $\Theta(r)$ against Radial Distance, $r$ with variation in Radiation Parameter, $N^{2}$.

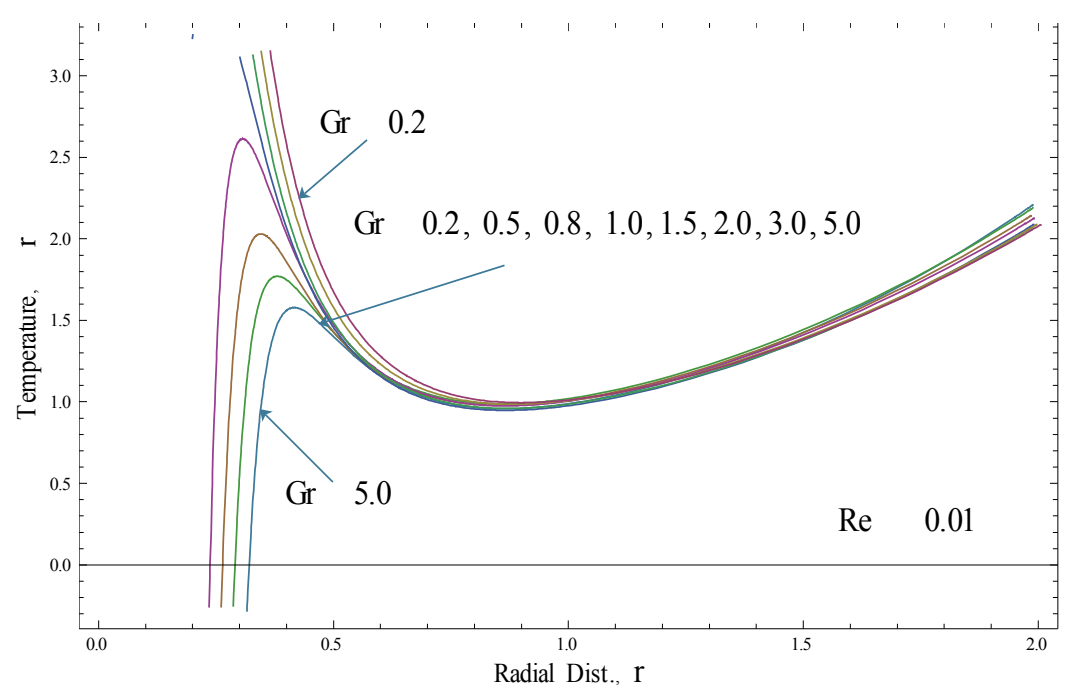

Figure 3. A Plot of Temperature, $\Theta(r)$ against Radial Distance, $r$ with variationin free convection parameter, Gr. 
The temperature reduction was as a result of loss of radiant energy emitted as gamma ray photons. However, just above the solar surface where the photons are released in the visible range as thermal radiation, the radiation parameter has a positive correlation with the plasma temperature. Similarly, the temperature decreases with increase in free convection parameter, Gr. Increase Gr, is seen to induce convective mixing of the plasma which leads to temperature reduction, though gradually near the solar surface.

Generally, the temperature profiles as established from the model was observed to decrease sharply from the core (at $r=$ 0.25 ) of the sphere to about, $r=0.7$ (i.e., $0.25 \leq r \leq 0.7$ ), but gradually from there to surface of the sphere, at $r=1.0$. The region within $0.25 \leq r \leq 0.7$ of the sphere corresponds to the radiation zone. Here high energy gamma ray photons are transmitted radiatively amidst scattering and absorption by plasma species. The drastic reduction in temperature was due to the interaction of the photons with the plasma species. While, within $r=0.7$ and $r=1.0$ corresponds to the convection zone. The high opacity in this region due to partially ionized heavy elements [20-22] renders radiation ineffective. Hence, the absorbed photons stir up the plasma to initiate convective motion. The convective mixing of the plasma accounts for the gradual decrease in temperature.

\section{Conclusion}

The thermal parameters of interest investigated in this study (that is, the radiation and free convection parameters) were seen to significantly influence the temperature distribution in the solar sphere. Both the radiation and free convection parameters are found to have negative correlation with the flow temperaturewithin the sphere. That is, increasing the radiation parameter, $N^{2}$ and free convection parameter, $G r$. led to a decrease in the plasma flow temperature in the sphere. Whereas, above the sphere (i.e. at radial distances, $r>1.0$ ) where the plasma density is sparse, increasingthe radiation and free convection parameters produced a corresponding increase in the plasma flow temperature. The decrease in the plasma flow temperature within the sphere with increase in the thermal parameters was observed to be more noticeable between radial distances, $r=$ 0.25 and $r=0.7$ (i.e., $0.25 \leq r \leq 0.7$; corresponding to the radiation zone of the solar interior) than between $r=0.7$ and $r=1.0$ (i.e., $0.7 \leq r \leq 1.0$; corresponding to the convection zone of the solar interior). This is attributable to the presence of partially ionized heavy elements in the convection zone which trap the high energy photons thereby reducing the rate of radiative heat loss.

\section{References}

[1] Alagoa, K. D., Tay, G. and Abbey, T. M. (1999). Radiative and free convective effects of a MHD flow through a porous medium between infinite parallel plates with time-dependent suction. Astrophysics and Space Science, 260: 455-468.
[2] Abbey, T. M. (2004). Effect of radiative heat transfer, free convection and time dependent suction on the flow of a twocomponent plasma in a porous medium sandwich between two infinite parallel plates. (Workshop proceedings) International Centre for Theoretical Physics, Trieste, Italy.

[3] Abbey, T. M. (2005). The flow of a two-component plasma past a rotating hot sphere with an oscillating diameter, workshop proceedings. (Workshop proceedings) International Centre for Theoretical Physics, Trieste, Italy.

[4] Roger, B. P. and Robert, C. J. (2016). Solar Physics and Terrestrial Effects: A Curriculum Guide for Teachers, Grade 7-12. (Third Edition), Space Weather Prediction Center, National Oceanic and Atmospheric Administration.

[5] Abbey, T. M., Bestman, A. R. and Mbeledogu, I. U. (1992). The flow of atwo-component plasma model in a porous rotating hot sphere. Astrophysics and Space Science, 197: 61-76.

[6] Abbey, T. M. and Bestman, A. R. (1995). Slip flow in a twocomponent plasma model with radiative heat transfer. International Journal of Energy Research, 19: 1-6.

[7] Abbey, T. M. (1996). The flow of a two-component plasma model past a rotating porous hot sphere. Nigerian Journal of Physics, 8S: 51-60.

[8] Sasikumar, J. and Govindarjan, A. (2016). Effect of heat and mass transfer on MHD oscillatory flow with chemical reaction and slip conditions in asymmetric wavy channel. Journal of Engineering and Applied Sciences, 11 (2): 11641170 .

[9] Sanatan, D., Mrinal, J. and Rabindra, N. J. (2011). Radiation effect on natural convection near a vertical plate embedded in porous medium with ramped wall temperature, Open $J$. of Fluid Dynamics, 1: 1-11.

[10] Priest, E. R. (1982). Solar magneto-hydrodynamics 1. Dordrecht, Holland: D. Reidel Publishing Company.

[11] Sherman, F. S. (1990). Viscous flow. (International Edition) New York City, NY: McGeaw-Hill. ISBN: 0-07056579-1.

[12] Alagoa, K. D. and Sakanaka, P. H. (1998). Gravitational stability of the solar plasma. International Centre for Theoretical Physics, Trieste, Italy. IC/98/112.

[13] Alagoa, K. D. and Abbey, T. M. (2001). Temperature distribution in the solar globe due to exponentially varying plasma density. Mathematical Science Forum, 3: 1-8.

[14] Abbey, T. M. and John, E. (2000). Transient Slip flow in a two-component plasma model with radiative heat transfer. Mathematical Science Forum, 2: 37-47.

[15] Bestman, A. R. (1983). Low Reynolds number flow in a heated tube of varying section. Journal of the Australian Mathematical Society Series, B25: 244-260.

[16] He, J.-H. (2006). Some asymptotic methods for strongly nonlinear equations. International Journal of Modern Physics B, 20 (10): 1141-1199.

[17] Biazar, J. and Eslami, M. (2011). A new homotopy perturbation method for solving systems of partial differential equations. Computers and Mathematics with Applications, 62: 225-234. 
[18] Molliq, Y. R. and Noorani, M. S. M. (2012). Solving the Fractional Rosenau-Hyman Equation via Variational Iteration Method and Homotopy Perturbation Method. International Journal of Differential Equations, 2012 (ID 472030): 1-14.

[19] Biazar, J. and Eslami, M. (2010). Analytic solutions for telegraph equation by differential transform method. Physics Letters A, 374: 2904-2906.

[20] Bailey, J. E., Rochau, G. A., Mancini, R. C., Iglesias, C. A., MacFarlane, J. J., Golovkin, I., Blancard, C., Colgan, J., Ph.
Cosse and Faussurier, G. (2009). Experimental investigation of opacity models for stellar interior, inertial fusion and high energy density plasmas. Physics of plasmas, 16: 058101.

[21] Hanasoge, S., Gizon, L. and Katepalli, R. S. (2015). Seismic Sounding of Convection in the Sun. Retrieved from: https://arxiv.org/pdf/1503.07961.pdf.

[22] Krief, M., Feigel, A. and Gazit, D. (2016). Line Broadening and the Solar Opacity Problem. Astrophysical Journal, 824: 98 (pp6). 